\title{
New Results for Generalized Gronwall Inequalities and Their Applications
}

\author{
Shi-you Lin \\ School of Mathematics and Statistics, Hainan Normal University, Haikou, Hainan 571158, China \\ Correspondence should be addressed to Shi-you Lin; linsyl111@aliyun.com \\ Received 1 May 2014; Accepted 5 June 2014; Published 19 June 2014 \\ Academic Editor: Hui-Sheng Ding
}

Copyright (c) 2014 Shi-you Lin. This is an open access article distributed under the Creative Commons Attribution License, which permits unrestricted use, distribution, and reproduction in any medium, provided the original work is properly cited.

In succession to our earlier work, we further provide some new generalized Gronwall inequalities and apply these inequalities to the study of qualitative estimations of solutions to certain fractional differential equations.

\section{Introduction}

It is well known that the Gronwall inequality contributes significantly to research on many differential and integral equations. An increasing number of generalizations of this inequality have been made in recent years to derive qualitative properties of solutions to various fractional differential equations. One remarkable result was obtained by Ye et al. in 2007 and is presented below.

Theorem 1 (see [1, Theorem 1]). For any $t \in[0, T)$,

$$
u(t) \leq a(t)+b(t) \int_{0}^{t}(t-s)^{\beta-1} u(s) d s
$$

where all functions are nonnegative and continuous. The constant $\beta>0 . b$ is a bounded and monotonically increasing function of $[0, T)$; then

$$
\begin{array}{r}
u(t) \leq a(t)+\int_{0}^{t}\left[\sum_{n=1}^{\infty} \frac{(b(t) \Gamma(\beta))^{n}}{\Gamma(n \beta)}(t-s)^{n \beta-1} a(s)\right] d s, \\
t \in[0, T) .
\end{array}
$$

This result continuously extends and improves and has been widely used in many studies (e.g., see [1-8]). Among them, our previous research [5] provides the following generalizations to deal with complex fractional differential equations.
Theorem 2 (see [5, Theorem 1.4]). For any $t \in[0, T)$,

$$
u(t) \leq a(t)+\sum_{i=1}^{n} b_{i}(t) \int_{0}^{t}(t-s)^{\beta_{i}-1} u(s) d s,
$$

where all functions are nonnegative and continuous. The constants $\beta_{i}>0 . b_{i}(i=1,2, \ldots, n)$ are bounded and monotonically increasing functions of $[0, T)$. Then

$$
\begin{array}{r}
u(t) \leq a(t) \\
+\sum_{k=1}^{\infty}\left(\sum_{1^{\prime}, 2^{\prime}, \ldots, k^{\prime}=1}^{n} \frac{\prod_{i=1}^{k}\left[b_{i^{\prime}}(t) \Gamma\left(\beta_{i^{\prime}}\right)\right]}{\Gamma\left(\sum_{i=1}^{k} \beta_{i^{\prime}}\right)}\right. \\
\left.\times \int_{0}^{t}(t-s)^{\sum_{i=1}^{k} \beta_{i^{\prime}}-1} a(s) d s\right), \\
t \in[0, T) .
\end{array}
$$

Theorem 3 (see [5, Theorem 1.5]). For any $t \in[1, T$ ),

$$
u(t) \leq a(t)+\sum_{i=1}^{n} b_{i}(t) \int_{1}^{t}\left(\ln \frac{t}{s}\right)^{\beta_{i}-1} \frac{u(s)}{s} d s,
$$


where all functions are nonnegative and continuous. The constants $\beta_{i}>0 . b_{i}(i=1,2, \ldots, n)$ are bounded and monotonically increasing functions of $[1, T)$. Then

$$
\begin{aligned}
& u(t) \leq a(t) \\
&+\sum_{k=1}^{\infty}\left(\sum_{1^{\prime}, 2^{\prime}, \ldots, k^{\prime}=1}^{n} \frac{\prod_{i=1}^{k}\left[b_{i^{\prime}}(t) \Gamma\left(\beta_{i^{\prime}}\right)\right]}{\Gamma\left(\sum_{i=1}^{k} \beta_{i^{\prime}}\right)}\right. \\
&\left.\times \int_{1}^{t}\left[\left(\ln \frac{t}{s}\right)^{\sum_{i=1}^{k} \beta_{i^{\prime}}-1} a(s)\right] \frac{d s}{s}\right), \\
& t \in[1, T) .
\end{aligned}
$$

In this paper, we aim to discuss further issues by using the aforementioned conclusions and suitable analytical techniques according to the above facts. We will establish several new classes of generalized Gronwall inequalities in the next section. In the last section, we will qualitatively analyze certain fractional differential equations to highlight the applications of the inequalities.

\section{Main Results and Proofs}

We introduce the useful Young's inequality with $\epsilon>0$ (see [9, page 622]); that is, for any $a, b>0$ and $1<p, q<+\infty$, $1 / p+1 / q=1$

$$
a b \leq \epsilon a^{p}+C(\epsilon) b^{q}
$$

where $C(\epsilon)=(\epsilon p)^{-q / p} q^{-1}$.

The first main result and its proof procedure are presented as follows.

Theorem 4. For any $t \in[0, T)$,

$$
u(t) \leq a(t)+\sum_{i=1}^{n} b_{i}(t) \int_{0}^{t}(t-s)^{\beta_{i}-1} c_{i}(s) u^{\lambda_{i}}(s) d s
$$

where all functions are nonnegative and continuous. For any $i \in\{1,2, \ldots, n\}$, the constants $\beta_{i}>0$ and $0<\lambda_{i}<1$, and the function $b_{i}(t)$ is bounded and monotonically increasing on $[0, T)$. Then

$$
\begin{array}{r}
u(t) \leq \tilde{a}(t) \\
+\sum_{k=1}^{\infty}\left(\sum_{1^{\prime}, 2^{\prime}, \ldots, k^{\prime}=1}^{n} \frac{\prod_{i=1}^{k}\left[\widetilde{b}_{i^{\prime}}(t) \Gamma\left(\beta_{i^{\prime}}\right)\right]}{\Gamma\left(\sum_{i=1}^{k} \beta_{i^{\prime}}\right)}\right. \\
\left.\times \int_{0}^{t}(t-s)^{\sum_{i=1}^{k} \beta_{i^{\prime}-1}} \tilde{a}(s) d s\right), \\
t \in[0, T),
\end{array}
$$

where

$$
\begin{gathered}
\tilde{a}(t)=a(t)+\sum_{i=1}^{n}\left(1-\lambda_{i}\right)\left(\frac{\lambda_{i}}{\varepsilon}\right)^{\lambda_{i} /\left(1-\lambda_{i}\right)} b_{i}(t) \\
\times \int_{0}^{t}(t-s)^{\beta_{i}-1}\left[c_{i}(s)\right]^{1 /\left(1-\lambda_{i}\right)} d s \\
\widetilde{b}_{i}(t)=\varepsilon \cdot b_{i}(t) .
\end{gathered}
$$

Here $\varepsilon$ is an arbitrary given positive number.

Proof. By Young's inequality,

$$
c_{i}(s) u^{\lambda_{i}}(s)
$$

$$
\begin{aligned}
& \leq \varepsilon \cdot\left[u^{\lambda_{i}}(s)\right]^{1 / \lambda_{i}}+\left(\frac{\lambda_{i}}{\varepsilon}\right)^{\lambda_{i} /\left(1-\lambda_{i}\right)} \cdot\left(1-\lambda_{i}\right) \cdot\left[c_{i}(s)\right]^{1 /\left(1-\lambda_{i}\right)} \\
& =\varepsilon \cdot u(s)+\left(\frac{\lambda_{i}}{\varepsilon}\right)^{\lambda_{i} /\left(1-\lambda_{i}\right)} \cdot\left(1-\lambda_{i}\right) \cdot\left[c_{i}(s)\right]^{1 /\left(1-\lambda_{i}\right)}
\end{aligned}
$$

which implies that, for any $t \in[0, T)$,

$$
\begin{aligned}
u(t) \leq & a(t)+\sum_{i=1}^{n} b_{i}(t) \int_{0}^{t}(t-s)^{\beta_{i}-1} c_{i}(s) u^{\lambda_{i}}(s) d s \\
\leq & a(t)+\sum_{i=1}^{n} b_{i}(t) \\
& \times \int_{0}^{t}(t-s)^{\beta_{i}-1}\{\varepsilon \cdot u(s) \\
= & \left\{\left(\frac{\lambda_{i}}{\varepsilon}\right)^{\lambda_{i} /\left(1-\lambda_{i}\right)} \cdot\left(1-\lambda_{i}\right)\right. \\
& \left\{\begin{array}{c}
\left.a(t)+\sum_{i=1}^{n}\left(1-\lambda_{i}\right)\left(\frac{\lambda_{i}}{\varepsilon}\right)^{1 /\left(1-\lambda_{i}\right)}\right\} d s \\
\lambda_{i} /\left(1-\lambda_{i}\right) \\
b_{i}(t) \\
+\sum_{i=1}^{n} \varepsilon \cdot b_{i}(t) \int_{0}^{t}(t-s)^{\beta_{i}-1} u(s) d s \\
\tilde{a}(t)+\sum_{i=1}^{n} \tilde{b}_{i}(t) \int_{0}^{t}(t-s)^{\beta_{i}-1} u(s) d s .
\end{array}\right]
\end{aligned}
$$

We immediately derived estimation (9) by using Theorem 2. This estimation completes the proof of Theorem 4 .

Our second result can be proved in the same manner by applying Young's inequality and Theorem 3. The proof procedure is similar to that of Theorem 4 and is omitted here. 
Theorem 5. For any $t \in[1, T)$,

$$
u(t) \leq a(t)+\sum_{i=1}^{n} b_{i}(t) \int_{1}^{t}\left(\ln \frac{t}{s}\right)^{\beta_{i}-1} \frac{c_{i}(s) u^{\lambda_{i}}(s)}{s} d s
$$

where all functions are nonnegative and continuous. For any $i \in\{1,2, \ldots, n\}$, the constants $\beta_{i}>0$ and $0<\lambda_{i}<1$, and the function $b_{i}(t)$ is bounded and monotonically increasing on $[1, T)$. Then

$$
\begin{array}{r}
u(t) \leq \bar{a}(t) \\
+\sum_{k=1}^{\infty}\left(\sum_{1^{\prime}, 2^{\prime}, \ldots, k^{\prime}=1}^{n} \frac{\prod_{i=1}^{k}\left[\bar{b}_{i^{\prime}}(t) \Gamma\left(\beta_{i^{\prime}}\right)\right]}{\Gamma\left(\sum_{i=1}^{k} \beta_{i^{\prime}}\right)}\right. \\
\left.\times \int_{1}^{t}\left(\ln \frac{t}{s}\right)^{\sum_{i=1}^{k} \beta_{i^{\prime}}-1} \frac{\bar{a}(s)}{s} d s\right), \\
t \in[1, T),
\end{array}
$$

where

$$
\begin{gathered}
\bar{a}(t)=a(t)+\sum_{i=1}^{n}\left(1-\lambda_{i}\right)\left(\frac{\lambda_{i}}{\varepsilon}\right)^{\lambda_{i} /\left(1-\lambda_{i}\right)} b_{i}(t) \\
\times \int_{1}^{t}\left(\ln \frac{t}{s}\right)^{\beta_{i}-1}\left[c_{i}(s)\right]^{1 /\left(1-\lambda_{i}\right)} \frac{d s}{s} ; \\
\bar{b}_{i}(t)=\varepsilon \cdot b_{i}(t) .
\end{gathered}
$$

Here $\varepsilon$ is an arbitrary given positive number.

Evidently, the following two corollaries are directly obtained by using Theorems 4 and 5 when choosing $\beta_{i}=1$ for any $i \in\{1,2, \ldots, n\}$.

Corollary 6. For any $t \in[0, T)$,

$$
u(t) \leq a(t)+\sum_{i=1}^{n} b_{i}(t) \int_{0}^{t} c_{i}(s) u^{\lambda_{i}}(s) d s
$$

where all functions are nonnegative and continuous. For any $i \in\{1,2, \ldots, n\}$, the constant $0<\lambda_{i}<1$, and the function $b_{i}(t)$ is bounded and monotonically increasing on $[0, T)$. Then

$$
\begin{array}{r}
u(t) \leq \tilde{a}(t) \\
+\sum_{k=1}^{\infty}\left(\sum_{1^{\prime}, 2^{\prime}, \ldots, k^{\prime}=1}^{n} \frac{\prod_{i=1}^{k} \tilde{b}_{i^{\prime}}(t)}{k !}\right. \\
\left.\times \int_{0}^{t}(t-s)^{k-1} \tilde{a}(s) d s\right), \\
t \in[0, T),
\end{array}
$$

where

$$
\begin{gathered}
\tilde{a}(t)=a(t)+\sum_{i=1}^{n}\left(1-\lambda_{i}\right)\left(\frac{\lambda_{i}}{\varepsilon}\right)^{\lambda_{i} /\left(1-\lambda_{i}\right)} b_{i}(t) \\
\times \int_{0}^{t}\left[c_{i}(s)\right]^{1 /\left(1-\lambda_{i}\right)} d s ; \\
\tilde{b}_{i}(t)=\varepsilon \cdot b_{i}(t) .
\end{gathered}
$$

Here $\varepsilon$ is an arbitrary given positive number.

Corollary 7. For any $t \in[1, T)$,

$$
u(t) \leq a(t)+\sum_{i=1}^{n} b_{i}(t) \int_{1}^{t} \frac{c_{i}(s) u^{\lambda_{i}}(s)}{s} d s,
$$

where all functions are nonnegative and continuous. For any $i \in\{1,2, \ldots, n\}$, the constant $0<\lambda_{i}<1$, and the function $b_{i}(t)$ is bounded and monotonically increasing on $[1, T)$. Then

$$
u(t) \leq \bar{a}(t)
$$

$$
\begin{aligned}
&+\sum_{k=1}^{\infty}\left(\sum_{1^{\prime}, 2^{\prime}, \ldots, k^{\prime}=1}^{n} \frac{\prod_{i=1}^{k} \bar{b}_{i^{\prime}}(t)}{k !}\right. \\
&\left.\times \int_{1}^{t}\left[\left(\ln \frac{t}{s}\right)^{k-1} \bar{a}(s)\right] \frac{d s}{s}\right), \\
& t \in[1, T),
\end{aligned}
$$

where

$$
\begin{aligned}
\bar{a}(t)=a(t)+\sum_{i=1}^{n}\left(1-\lambda_{i}\right)\left(\frac{\lambda_{i}}{\varepsilon}\right)^{\lambda_{i} /\left(1-\lambda_{i}\right)} b_{i}(t) \\
\times \int_{1}^{t}\left[c_{i}(s)\right]^{1 /\left(1-\lambda_{i}\right)} \frac{d s}{s} ; \\
\bar{b}_{i}(t)=\varepsilon \cdot b_{i}(t) .
\end{aligned}
$$

Here $\varepsilon$ is an arbitrary given positive number.

Our next task is estimating the nonnegative and continuous function $u(t)$, which satisfies, for any $t \in[0, T)$,

$$
\begin{aligned}
u(t) \leq & a(t)+b(t) \int_{0}^{t} c(s) u(s) d s \\
& +\sum_{i=1}^{n} b_{i}(t) \int_{0}^{t}(t-s)^{\beta_{i}-1} c_{i}(s) u^{\lambda_{i}}(s) d s
\end{aligned}
$$

where all the functions are nonnegative and continuous. For any $i \in\{1,2, \ldots, n\}$, the constants $\beta_{i}>0$ and $0<\lambda_{i}<1$, and the functions $b(t)$ and $b_{i}(t)$ are bounded and monotonically increasing on $[0, T)$. Put

$$
M_{i}(t)=\max _{0 \leq s \leq t}\left\{b_{i}(s) c(s)\right\} .
$$


Suppose that

$$
\begin{gathered}
A(t)=a(t)+b(t) \int_{0}^{t} c(s) e^{\int_{s}^{t} c(w) b(w) d w} \cdot a(s) d s \\
B_{i}(t)=b_{i}(t)+\frac{t \cdot b(t) \cdot M_{i}(t)}{\beta_{i}} e^{\int_{0}^{t} c(w) b(w) d w}, \quad i=1,2, \ldots, n .
\end{gathered}
$$

Obviously, $B_{i}(t) \geq 0(i=1,2, \ldots, n)$ are bounded and monotonically increasing functions. Moreover, we assumed that

$$
\begin{gathered}
\widetilde{A}(t)=A(t)+\sum_{i=1}^{n}\left(1-\lambda_{i}\right)\left(\frac{\lambda_{i}}{\varepsilon}\right)^{\lambda_{i} /\left(1-\lambda_{i}\right)} B_{i}(t) \\
\times \int_{0}^{t}(t-s)^{\beta_{i}-1}\left[c_{i}(s)\right]^{1 /\left(1-\lambda_{i}\right)} d s ; \\
\widetilde{B}_{i}(t)=\varepsilon B_{i}(t), \quad i=1,2, \ldots, n .
\end{gathered}
$$

Here $\varepsilon$ is an arbitrary given positive number. The third main result is given as follows.

Theorem 8. For any $t \in[0, T)$, the nonnegative and continuous function $u(t)$ satisfies the inequality (22); then

$$
\begin{array}{r}
u(t) \leq \widetilde{A}(t) \\
+\sum_{k=1}^{\infty}\left(\sum_{1^{\prime}, 2^{\prime}, \ldots, k^{\prime}=1}^{n} \frac{\prod_{i=1}^{k}\left[\widetilde{B}_{i^{\prime}}(t) \Gamma\left(\beta_{i^{\prime}}\right)\right]}{\Gamma\left(\sum_{i=1}^{k} \beta_{i^{\prime}}\right)}\right. \\
\left.\times \int_{0}^{t}(t-s)^{\sum_{i=1}^{k} \beta_{i^{\prime}}-1} \widetilde{A}(s) d s\right), \\
t \in[0, T),
\end{array}
$$

where the expressions $\widetilde{A}(t)$ and $\widetilde{B}_{i}(t)$ are described in (25).

Proof. Suppose that

$$
I(t)=a(t)+\sum_{i=1}^{n} b_{i}(t) \int_{0}^{t}(t-s)^{\beta_{i}-1} c_{i}(s) u^{\lambda_{i}}(s) d s .
$$

Then, (22) transforms into the following form:

$$
u(t) \leq I(t)+b(t) \int_{0}^{t} c(s) u(s) d s
$$

Therefore,

$$
c(t) u(t) \leq c(t) I(t)+c(t) b(t) \int_{0}^{t} c(s) u(s) d s .
$$

Letting $u_{1}(t)=c(t) u(t), I_{1}(t)=c(t) I(t)$, and $J_{1}(t)=c(t) b(t)$ obtains

$$
u_{1}(t) \leq I_{1}(t)+J_{1}(t) \int_{0}^{t} u_{1}(s) d s
$$

By using the classical Gronwall inequality (see [10, page 15]), we have

$$
u_{1}(t) \leq I_{1}(t)+J_{1}(t) \int_{0}^{t} I_{1}(s) e^{\int_{s}^{t} J_{1}(w) d w} d s
$$

that is,

$$
\begin{aligned}
u(t) \leq & I(t)+b(t) \int_{0}^{t} c(s) I(s) e^{\int_{s}^{t} c(w) b(w) d w} d s \\
= & {\left[a(t)+b(t) \int_{0}^{t} c(s) a(s) e^{\int_{s}^{t} c(w) b(w) d w} d s\right] } \\
& +\sum_{i=1}^{n} b_{i}(t) \int_{0}^{t}(t-s)^{\beta_{i}-1} c_{i}(s) u^{\lambda_{i}}(s) d s \\
& +b(t) \sum_{i=1}^{n} \int_{0}^{t} \int_{0}^{s} c(s) e^{\int_{s}^{t} c(w) b(w) d w} \\
& \quad \times b_{i}(s)(s-v)^{\beta_{i}-1} c_{i}(v) u^{\lambda_{i}}(v) d v d s
\end{aligned}
$$

given that

$$
\begin{aligned}
& \sum_{i=1}^{n} \int_{0}^{t} \int_{0}^{s} b_{i}(s) c(s) e^{\int_{s}^{t} c(w) b(w) d w} \\
& \quad \times(s-v)^{\beta_{i}-1} c_{i}(v) u^{\lambda_{i}}(v) d v d s \\
& =\sum_{i=1}^{n} \int_{0}^{t} \int_{v}^{t} b_{i}(s) c(s) e^{\int_{s}^{t} c(w) b(w) d w} \\
& \times(s-v)^{\beta_{i}-1} c_{i}(v) u^{\lambda_{i}}(v) d s d v \\
& \leq e^{\int_{0}^{t} c(w) b(w) d w} \\
& \times \sum_{i=1}^{n} M_{i}(t) \int_{0}^{t} \int_{v}^{t}(s-v)^{\beta_{i}-1} c_{i}(v) u^{\lambda_{i}}(v) d s d v \\
& =e^{\int_{0}^{t} c(w) b(w) d w} \sum_{i=1}^{n} \frac{1}{\beta_{i}} M_{i}(t) \\
& \times \int_{i=1}^{t}(t-v)^{\beta_{i}-1}(t-v) c_{i}(v) u^{\lambda_{i}}(v) d v \\
& \leq e^{\int_{0}^{t} c(w) b(w) d w \int_{0}^{n}} \frac{1}{\beta_{i}} t M_{i}(t) \\
& \times \int^{\beta_{i}-1} c_{i}(v) u^{\lambda_{i}}(v) d v,
\end{aligned}
$$

where the function $M_{i}(t)$ is defined in (23). Combining (24), (32), and (33) yields the following inequality:

$$
u(t) \leq A(t)+\sum_{i=1}^{n} B_{i}(t) \int_{0}^{t}(t-s)^{\beta_{i}-1} c_{i}(s) u^{\lambda_{i}}(s) d s .
$$

The estimation (26) is obtained according to Theorem 4 . This process completes the proof of Theorem 8 . 
In the same manner, the final result in this section can be obtained by applying the conclusion of Theorem 5 .

Theorem 9. For any $t \in[1, T)$,

$$
\begin{aligned}
u(t) \leq & a(t)+b(t) \int_{1}^{t} c(s) u(s) \frac{d s}{s} \\
& +\sum_{i=1}^{n} b_{i}(t) \int_{1}^{t}\left(\ln \frac{t}{s}\right)^{\beta_{i}-1} c_{i}(s) u^{\lambda_{i}}(s) \frac{d s}{s},
\end{aligned}
$$

where all functions are nonnegative and continuous. For any $i \in\{1,2, \ldots, n\}$, the constants $\beta_{i}>0$ and $0<\lambda_{i}<1$, and the functions $b(t)$ and $b_{i}(t)$ are bounded and monotonically increasing on $[1, T)$. Then

$$
\begin{array}{r}
u(t) \leq \bar{A}(t) \\
+\sum_{k=1}^{\infty}\left(\sum_{1^{\prime}, 2^{\prime}, \ldots, k^{\prime}=1}^{n} \frac{\prod_{i=1}^{k}\left[\bar{B}_{i^{\prime}}(t) \Gamma\left(\beta_{i^{\prime}}\right)\right]}{\Gamma\left(\sum_{i=1}^{k} \beta_{i^{\prime}}\right)}\right. \\
\left.\times \int_{1}^{t}\left(\ln \frac{t}{s}\right)^{\sum_{i=1}^{k} \beta_{i^{\prime}}-1} \frac{\bar{A}(s)}{s} d s\right), \\
t \in[1, T),
\end{array}
$$

where

$$
\begin{gathered}
\bar{A}(t)=A^{\prime}(t)+\sum_{i=1}^{n}\left(1-\lambda_{i}\right)\left(\frac{\lambda_{i}}{\varepsilon}\right)^{\lambda_{i} /\left(1-\lambda_{i}\right)} B_{i}^{\prime}(t) \\
\times \int_{1}^{t}\left(\ln \frac{t}{s}\right)^{\beta_{i}-1}\left[c_{i}(s)\right]^{1 /\left(1-\lambda_{i}\right)} \frac{d s}{s} ; \\
\bar{B}_{i}(t)=\varepsilon \cdot B_{i}^{\prime}(t) .
\end{gathered}
$$

Here

$$
\begin{array}{r}
A^{\prime}(t)=a(t)+b(t) \int_{1}^{t} c(s) e^{\int_{s}^{t} c(w) b(w)(d w / w)} \cdot a(s) \frac{d s}{s} \\
B_{i}^{\prime}(t)=b_{i}(t)+\frac{\ln t \cdot b(t) \cdot M_{i}^{\prime}(t)}{\beta_{i}} e^{\int_{1}^{t} c(w) b(w)(d w / w)} \\
i=1,2, \ldots, n .
\end{array}
$$

Also $M_{i}^{\prime}(t)=\max _{1 \leq s \leq t}\left\{b_{i}(s) c(s)\right\}$, and $\varepsilon$ is an arbitrary given positive number.

The proof procedure of Theorem 9 is relatively similar to that of Theorem 8 . Hence, the procedure will not be presented in this paper.

\section{Applications}

In this section, we apply the main results in Section 2 to provide qualitative conclusions for solutions of certain fractional differential equations. First, the definitions and some properties of the Riemann-Liouville fractional derivative and integral need to be recalled.
Definition 10 (see [11-16]). For any $0<\beta<1$ and a continuous function $w$, the $\beta$ th Riemann-Liouville type fractional order derivative $D_{R}^{\beta} w$ and the corresponding fractional integral operator $I_{R}^{\beta} w$ are defined by

$$
\begin{gathered}
I_{R}^{\beta} w(t)=\frac{1}{\Gamma(\beta)} \int_{0}^{t}(t-s)^{\beta-1} w(s) d s ; \\
D_{R}^{\beta} w(t)=\frac{1}{\Gamma(1-\beta)} \frac{d}{d t} \int_{0}^{t}(t-s)^{-\beta} w(s) d s .
\end{gathered}
$$

Lemma 11 (see [13, Lemma 2.2]). For any $0<\beta<1$ and $a$ continuous function $w$,

$$
I_{R}^{\beta} D_{R}^{\beta} w(t)=w(t)+k \cdot t^{\beta-1}
$$

where $k$ is a certain constant in $\mathbb{R}$.

Lemma 12 (see [17, Page 14]). For any $\alpha, \beta>0$ and $a$ continuous function $w$,

$$
\begin{gathered}
I_{R}^{\alpha} I_{R}^{\beta} w(t)=I_{R}^{\alpha+\beta} w(t) \\
I_{R}^{\alpha} t^{\beta}=\frac{\Gamma(\beta+1)}{\Gamma(\alpha+\beta+1)} t^{\alpha+\beta} .
\end{gathered}
$$

Given the aforementioned preliminary knowledge, we consider the following initial value problem:

$$
\begin{aligned}
& \sum_{i=1}^{n} D_{R}^{\beta_{n+1}-\beta_{i}}\left[c_{i}(t) u^{\lambda_{i}}(t)\right]+D_{R}^{\beta_{n+1}}\left[u^{\lambda_{0}}(t)\right]=f(t, u(t)) \\
& \left.\left\{\sum_{i=1}^{n} I_{R}^{1+\beta_{i}-\beta_{n+1}}\left[c_{i}(t) u^{\lambda_{i}}(t)\right]+I_{R}^{1-\beta_{n+1}}\left[u^{\lambda_{0}}(t)\right]\right\}\right|_{t=0}=\delta,
\end{aligned}
$$

where all functions are continuous. Moreover $c_{i}(t)>0$ and the constants $\lambda_{i}, \beta_{i}>0(i=1,2, \ldots, n)$. Consider $\lambda_{0}>0$, $\delta \in \mathbb{R}$, and $1>\beta_{n+1}>\max _{1 \leq i \leq n}\left\{\beta_{i}\right\}$.

Theorem 13. Suppose that, for any $t \in[0, T]$ and $y, z \in \mathbb{R}$,

$$
|f(t, y)-f(t, z)| \leq c_{n+1}(t)\left|y^{\lambda_{n+1}}-z^{\lambda_{n+1}}\right|,
$$

where the function $c_{n+1}(t)>0$ is continuous and the constant $\lambda_{n+1} \in(0,1)$. 
(i) If $\lambda_{0}>\max _{1 \leq i \leq n+1}\left\{\lambda_{i}\right\}$, then for any solution $u(t)$ of the problem (42),

$|u(t)|$

$\leq\left\{\widetilde{A}_{0}(t)\right.$

$$
+\sum_{k=1}^{\infty}\left(\sum_{1^{\prime}, 2^{\prime}, \ldots, k^{\prime}=1}^{n+1} \frac{\varepsilon^{k}}{\Gamma\left(\sum_{i=1}^{k} \beta_{i^{\prime}}\right)}\right.
$$

$$
\left.\left.\times \int_{0}^{t}(t-s)^{\sum_{i=1}^{k} \beta_{i^{\prime}}-1} \widetilde{A}_{0}(s) d s\right)\right\}^{1 / \lambda_{0}},
$$

where

$$
\begin{gathered}
\widetilde{A}_{0}(t)=\frac{|\delta| t^{\beta_{n+1}-1}}{\Gamma\left(\beta_{n+1}\right)}+\frac{1}{\Gamma\left(\beta_{n+1}\right)} \int_{0}^{t}(t-s)^{\beta_{n+1}-1}|f(s, 0)| d s \\
+\sum_{i=1}^{n+1} \frac{1}{\Gamma\left(\beta_{i}\right)}\left(1-\frac{\lambda_{i}}{\lambda_{0}}\right)\left(\frac{\lambda_{i}}{\varepsilon \lambda_{0}}\right)^{\lambda_{i} /\left(\lambda_{0}-\lambda_{i}\right)} \\
\times \int_{0}^{t}(t-s)^{\beta_{i}-1}\left[c_{i}(s)\right]^{\lambda_{0} /\left(\lambda_{0}-\lambda_{i}\right)} d s .
\end{gathered}
$$

Here $\varepsilon$ is an arbitrary given positive number.

(ii) If $\lambda_{0} \leq \min _{1 \leq i \leq n+1}\left\{\lambda_{i}\right\}$, then the continuous solution of problem (42) is unique.

Proof. Since $1>\beta_{n+1}>\max _{1 \leq i \leq n}\left\{\beta_{i}\right\}$, we obtain the following by using Lemmas 11 and 12:

$$
\begin{aligned}
I_{R}^{\beta_{n+1}} f(t, u(t)) & \\
= & I_{R}^{\beta_{n+1}} D_{R}^{\beta_{n+1}}\left[u^{\lambda_{0}}(t)\right] \\
& +\sum_{i=1}^{n} I_{R}^{\beta_{n+1}} D_{R}^{\beta_{n+1}-\beta_{i}}\left[c_{i}(t) u^{\lambda_{i}}(t)\right] \\
= & I_{R}^{\beta_{n+1}} D_{R}^{\beta_{n+1}}\left[u^{\lambda_{0}}(t)\right] \\
& +\sum_{i=1}^{n} I_{R}^{\beta_{i}}\left(I_{R}^{\beta_{n+1}-\beta_{i}} D_{R}^{\beta_{n+1}-\beta_{i}}\left[c_{i}(t) u^{\lambda_{i}}(t)\right]\right) \\
= & u^{\lambda_{0}}(t)+k_{n+1} t^{\beta_{n+1}-1} \\
& +\sum_{i=1}^{n} I_{R}^{\beta_{i}}\left(c_{i}(t) u^{\lambda_{i}}(t)+k_{i} t^{\beta_{n+1}-\beta_{i}-1}\right)
\end{aligned}
$$

$$
\begin{aligned}
= & u^{\lambda_{0}}(t)+\sum_{i=1}^{n} I_{R}^{\beta_{i}}\left[c_{i}(t) u^{\lambda_{i}}(t)\right] \\
& +\frac{t^{\beta_{n+1}-1}}{\Gamma\left(\beta_{n+1}\right)}\left\{k_{n+1} \Gamma\left(\beta_{n+1}\right)+\sum_{i=1}^{n} k_{i} \Gamma\left(\beta_{n+1}-\beta_{i}\right)\right\},
\end{aligned}
$$

where $k_{i}, i=1,2, \ldots, n+1$, are some constants. Therefore,

$$
\begin{aligned}
0 & =\left.\left\{\int_{0}^{t} f(s, u(s)) d s\right\}\right|_{t=0} \\
& =\left.\left\{I_{R}^{1-\beta_{n+1}} I_{R}^{\beta_{n+1}} f(t, u(t))\right\}\right|_{t=0} \\
& =\left\{\sum_{i=1}^{n} I_{R}^{1+\beta_{i}-\beta_{n+1}}\left[c_{i}(t) u^{\lambda_{i}}(t)\right]\right.
\end{aligned}
$$

$$
\begin{gathered}
\left.+I_{R}^{1-\beta_{n+1}}\left[u^{\lambda_{0}}(t)\right]\right\}\left.\right|_{t=0} \\
+k_{n+1} \Gamma\left(\beta_{n+1}\right)+\sum_{i=1}^{n} k_{i} \Gamma\left(\beta_{n+1}-\beta_{i}\right) \\
=\delta+k_{n+1} \Gamma\left(\beta_{n+1}\right)+\sum_{i=1}^{n} k_{i} \Gamma\left(\beta_{n+1}-\beta_{i}\right) ;
\end{gathered}
$$

that is,

$$
k_{n+1} \Gamma\left(\beta_{n+1}\right)+\sum_{i=1}^{n} k_{i} \Gamma\left(\beta_{n+1}-\beta_{i}\right)=-\delta .
$$

Integrating this equality into (46) obtains

$$
\begin{aligned}
u^{\lambda_{0}}(t) & \\
= & I_{R}^{\beta_{n+1}} f(t, u(t))-\sum_{i=1}^{n} I_{R}^{\beta_{i}}\left[c_{i}(t) u^{\lambda_{i}}(t)\right]+\frac{\delta t^{\beta_{n+1}-1}}{\Gamma\left(\beta_{n+1}\right)} \\
= & \frac{1}{\Gamma\left(\beta_{n+1}\right)} \int_{0}^{t}(t-s)^{\beta_{n+1}-1} f(s, u(s)) d s+\frac{\delta t^{\beta_{n+1}-1}}{\Gamma\left(\beta_{n+1}\right)} \\
& -\sum_{i=1}^{n} \frac{1}{\Gamma\left(\beta_{i}\right)} \int_{0}^{t}(t-s)^{\beta_{i}-1} c_{i}(s) u^{\lambda_{i}}(s) d s .
\end{aligned}
$$

Let $U(t)=u^{\lambda_{0}}(t)$, and applying (49), given the fact that

$$
\begin{aligned}
|f(s, u(s))| & \leq|f(s, 0)|+|f(s, u(s))-f(s, 0)| \\
& \leq|f(s, 0)|+c_{n+1}(s)|u(s)|^{\lambda_{n+1}},
\end{aligned}
$$


obtains the following:

$$
\begin{aligned}
|U(t)|= & |u(t)|^{\lambda_{0}} \\
\leq & \frac{1}{\Gamma\left(\beta_{n+1}\right)} \int_{0}^{t}(t-s)^{\beta_{n+1}-1}|f(s, u(s))| d s+\frac{|\delta| t^{\beta_{n+1}-1}}{\Gamma\left(\beta_{n+1}\right)} \\
& +\sum_{i=1}^{n} \frac{1}{\Gamma\left(\beta_{i}\right)} \int_{0}^{t}(t-s)^{\beta_{i}-1} c_{i}(s)|u(s)|^{\lambda_{i}} d s \\
\leq & \frac{1}{\Gamma\left(\beta_{n+1}\right)} \int_{0}^{t}(t-s)^{\beta_{n+1}-1}|f(s, 0)| d s+\frac{|\delta| t^{\beta_{n+1}-1}}{\Gamma\left(\beta_{n+1}\right)} \\
& +\sum_{i=1}^{n+1} \frac{1}{\Gamma\left(\beta_{i}\right)} \int_{0}^{t}(t-s)^{\beta_{i}-1} c_{i}(s)|u(s)|^{\lambda_{i}} d s \\
= & \left\{\frac{1}{\Gamma\left(\beta_{n+1}\right)} \int_{0}^{t}(t-s)^{\beta_{n+1}-1}|f(s, 0)| d s+\frac{|\delta| t^{\beta_{n+1}-1}}{\Gamma\left(\beta_{n+1}\right)}\right\} \\
& +\sum_{i=1}^{n+1} \frac{1}{\Gamma\left(\beta_{i}\right)} \int_{0}^{t}(t-s)^{\beta_{i}-1} c_{i}(s)|U(s)|^{\lambda_{i} / \lambda_{0}} d s .
\end{aligned}
$$

If $\lambda_{0}>\max _{1 \leq i \leq n+1}\left\{\lambda_{i}\right\}$, then, for any $i \in\{1,2, \ldots, n+1\}, 0<$ $\lambda_{i} / \lambda_{0}<1$. According to Theorem 4 , for any $t \in[0, T]$,

$$
\begin{aligned}
|U(t)| \leq \widetilde{A}_{0}(t) & \\
& +\sum_{k=1}^{\infty}\left(\sum_{1^{\prime}, 2^{\prime}, \ldots, k^{\prime}=1}^{n+1} \frac{\varepsilon^{k}}{\Gamma\left(\sum_{i=1}^{k} \beta_{i^{\prime}}\right)}\right. \\
& \left.\times \int_{0}^{t}(t-s)^{\sum_{i=1}^{k} \beta_{i^{\prime}}-1} \widetilde{A}_{0}(s) d s\right),
\end{aligned}
$$

where the expression of $\widetilde{A}_{0}(t)$ is shown in (45). Hence, the conclusion of (i) is derived.

In proving (ii), we assume that problem (42) has two continuous solutions $u$ and $v$. Combining with the fact that $c_{i}(t) \in C[0, T]$ for any $1 \leq i \leq n+1$ and the boundedness of the continuous function on a closed interval, there exists a finite number $M$ which satisfies that, for any $t \in[0, T]$,

$$
M>\max \left\{|u(t)|,|v(t)|, \max _{1 \leq i \leq n+1}\left|c_{i}(t)\right|\right\} .
$$

Cauchy's mean value theorem provides

$$
\begin{aligned}
\left|u^{\lambda_{i}}(t)-v^{\lambda_{i}}(t)\right| & =\left|u^{\lambda_{0}}(t)-v^{\lambda_{0}}(t)\right| \cdot\left|\frac{\lambda_{i} \cdot \xi_{i}^{\lambda_{i}-1}}{\lambda_{0} \cdot \xi_{i}^{\lambda_{0}-1}}\right| \\
& =\frac{\lambda_{i}}{\lambda_{0}}\left|u^{\lambda_{0}}(t)-v^{\lambda_{0}}(t)\right| \cdot\left|\xi_{i}\right|^{\lambda_{i}-\lambda_{0}}
\end{aligned}
$$

where $\xi_{i}, i=1,2, \ldots, n+1$, are the numbers between $u(t)$ and $v(t)$. The following estimation is deduced by applying (53) and the hypothesis of $\lambda_{0} \leq \min _{1 \leq i \leq n+1}\left\{\lambda_{i}\right\}$ in (ii):

$$
\left|u^{\lambda_{i}}(t)-v^{\lambda_{i}}(t)\right| \leq \frac{\lambda_{i}}{\lambda_{0}}\left|u^{\lambda_{0}}(t)-v^{\lambda_{0}}(t)\right| \cdot M^{\lambda_{i}-\lambda_{0}}
$$

holds for any $t \in[0, T]$ and $i=1,2, \ldots, n+1$. Therefore, (49), (53), and (55) obtain

$$
\begin{aligned}
& \left|u^{\lambda_{0}}(t)-v^{\lambda_{0}}(t)\right| \\
& =\mid \frac{1}{\Gamma\left(\beta_{n+1}\right)} \int_{0}^{t}(t-s)^{\beta_{n+1}-1} \\
& \quad \times[f(s, u(s))-f(s, v(s))] d s \\
& \quad-\sum_{i=1}^{n} \frac{1}{\Gamma\left(\beta_{i}\right)} \int_{0}^{t}(t-s)^{\beta_{i}-1} c_{i}(s) \\
& \quad \times\left[u^{\lambda_{i}}(s)-v^{\lambda_{i}}(s)\right] d s \mid \\
& \leq \sum_{i=1}^{n+1} \frac{1}{\Gamma\left(\beta_{i}\right)} \int_{0}^{t}(t-s)^{\beta_{i}-1} \\
& \times c_{i}(s)\left|u^{\lambda_{i}}(s)-v^{\lambda_{i}}(s)\right| d s \\
& \leq \sum_{i=1}^{n+1} \frac{\lambda_{i} M^{1+\lambda_{i}-\lambda_{0}}}{\lambda_{0} \Gamma\left(\beta_{i}\right)} \int_{0}^{t}(t-s)^{\beta_{i}-1} \\
& \times\left|u^{\lambda_{0}}(s)-v^{\lambda_{0}}(s)\right| d s .
\end{aligned}
$$

According to Theorem 2,

$$
\left|u^{\lambda_{0}}(t)-v^{\lambda_{0}}(t)\right| \leq 0,
$$

which means that

$$
u(t)=v(t), \quad t \in[0, T] .
$$

This completes the proof of (ii).

Moreover, we can also address the following initial value problem with the Hadamard type fractional derivative:

$$
\begin{aligned}
& \sum_{i=1}^{n} D_{H}^{\alpha_{n+1}-\alpha_{i}}\left[d_{i}(t) u^{\gamma_{i}}(t)\right]+D_{H}^{\alpha_{n+1}}\left[u^{\gamma_{0}}(t)\right]=g(t, u(t)) \\
& \left.\left\{\sum_{i=1}^{n} I_{H}^{1+\alpha_{i}-\alpha_{n+1}}\left[d_{i}(t) u^{\gamma_{i}}(t)\right]+I_{H}^{1-\alpha_{n+1}}\left[u^{\gamma_{0}}(t)\right]\right\}\right|_{t=1}=\eta
\end{aligned}
$$

where all functions are continuous. $d_{i}(t)>0$ and the constants $\gamma_{i}, \alpha_{i}>0(i=1,2, \ldots, n)$. Also $\gamma_{0}>0, \eta \in \mathbb{R}$, and $1>\alpha_{n+1}>\max _{1 \leq i \leq n}\left\{\alpha_{i}\right\}$. For any $\alpha \in(0,1)$ and a continuous function $w$, the operators $D_{H}^{\alpha}$ and $I_{H}^{\alpha}$ are presented below (see [18, page 110$])$ :

$$
\begin{gathered}
D_{H}^{\alpha} w(t)=\frac{1}{\Gamma(1-\alpha)}\left(t \frac{d}{d t}\right) \int_{1}^{t}\left(\ln \frac{t}{s}\right)^{-\alpha} \frac{w(s)}{s} d s ; \\
I_{H}^{\alpha} w(t)=\frac{1}{\Gamma(\alpha)} \int_{1}^{t}\left(\ln \frac{t}{s}\right)^{\alpha-1} \frac{w(s)}{s} d s .
\end{gathered}
$$

We are able to deduce the following result by Theorems 3 and 5. 
Theorem 14. Suppose that, for any $t \in[1, T]$ and $y, z \in \mathbb{R}$, $|g(t, y)-g(t, z)| \leq d_{n+1}(t)\left|y^{\gamma_{n+1}}-z^{\gamma_{n+1}}\right|$, where the function $d_{n+1}(t)>0$ is continuous and the constant $\gamma_{n+1} \in(0,1)$.

(i) If $\gamma_{0}>\max _{1 \leq i \leq n+1}\left\{\gamma_{i}\right\}$, then, for any solution $u(t)$ of problem (59),

$$
\begin{array}{r}
|u(t)| \\
\leq\left\{\bar{A}_{0}(t)+\sum_{k=1}^{\infty}\left(\sum_{1^{\prime}, 2^{\prime}, \ldots, k^{\prime}=1}^{n+1} \frac{\varepsilon^{k}}{\Gamma\left(\sum_{i=1}^{k} \alpha_{i^{\prime}}\right)}\right.\right. \\
\left.\left.\times \int_{1}^{t}\left(\ln \frac{t}{s}\right)^{\sum_{i=1}^{k}} \bar{A}_{0}(s) \frac{d s}{s}\right)\right\}^{1 / \gamma_{0}}, \\
t \in[1, T],
\end{array}
$$

where

$$
\begin{aligned}
& \bar{A}_{0}(t) \\
& =\frac{|\eta|(\ln t)^{\alpha_{n+1}-1}}{\Gamma\left(\alpha_{n+1}\right)} \\
& +\frac{1}{\Gamma\left(\alpha_{n+1}\right)} \int_{1}^{t}\left(\ln \frac{t}{s}\right)^{\alpha_{n+1}-1}|g(s, 0)| \frac{d s}{s} \\
& +\sum_{i=1}^{n+1} \frac{1}{\Gamma\left(\alpha_{i}\right)}\left(1-\frac{\gamma_{i}}{\gamma_{0}}\right)\left(\frac{\gamma_{i}}{\varepsilon \gamma_{0}}\right)^{\gamma_{i} /\left(\gamma_{0}-\gamma_{i}\right)} \\
& \quad \times \int_{1}^{t}\left(\ln \frac{t}{s}\right)^{\alpha_{i}-1}\left[d_{i}(s)\right]^{\gamma_{0} /\left(\gamma_{0}-\gamma_{i}\right)} \frac{d s}{s} .
\end{aligned}
$$

Here $\varepsilon$ is an arbitrary given positive number.

(ii) If $\gamma_{0} \leq \min _{1 \leq i \leq n+1}\left\{\gamma_{i}\right\}$, then the continuous solution of problem (59) is unique.

The proof procedure is similar to that of Theorem 13 . Thus, the procedure is omitted here.

\section{Conflict of Interests}

The author declares that there is no conflict of interests regarding the publication of this paper.

\section{Acknowledgments}

The author would like to thank the referees for their detailed and helpful suggestions for revising this paper. This work was supported by the National Natural Science Foundation of China (Grant nos. 11226167 and 11361020).

\section{References}

[1] H. Ye, J. Gao, and Y. Ding, "A generalized Gronwall inequality and its application to a fractional differential equation," Journal of Mathematical Analysis and Applications, vol. 328, no. 2, pp. 1075-1081, 2007.
[2] A. Abdeldaim and M. Yakout, "On some new integral inequalities of Gronwall-Bellman-Pachpatte type," Applied Mathematics and Computation, vol. 217, no. 20, pp. 7887-7899, 2011.

[3] Q. Feng and F. Meng, "Some new Gronwall-type inequalities arising in the research of fractional differential equations," Journal of Inequalities and Applications, vol. 2013, article 429, 2013.

[4] Q. Feng and B. Zheng, "Generalized Gronwall-Bellman-type delay dynamic inequalities on time scales and their applications," Applied Mathematics and Computation, vol. 218, no. 15, pp. 7880-7892, 2012.

[5] S. Lin, "Generalized Gronwall inequalities and their applications to fractional differential equations," Journal of Inequalities and Applications, vol. 2013, article 549, 2013.

[6] L. Li, F. Meng, and L. He, "Some generalized integral inequalities and their applications," Journal of Mathematical Analysis and Applications, vol. 372, no. 1, pp. 339-349, 2010.

[7] J. Shao and F. Meng, "Gronwall-Bellman type inequalities and their applications to fractional differential equations," Abstract and Applied Analysis, vol. 2013, Article ID 217641, 7 pages, 2013.

[8] H. Ye and J. Gao, "Henry-Gronwall type retarded integral inequalities and their applications to fractional differential equations with delay," Applied Mathematics and Computation, vol. 218, no. 8, pp. 4152-4160, 2011.

[9] L. C. Evans, Partial Differential Equations, vol. 19 of Graduate Studies in Mathematics, American Mathematical Society, Providence, RI, USA, 1998.

[10] C. Corduneanu, Principles of Differential and Integral Equations, Allyn and Bacon, Boston, Mass, USA, 1971.

[11] B. Ahmad, S. K. Ntouyas, and A. Alsaedi, "New existence results for nonlinear fractional differential equations with threepoint integral boundary conditions," Advances in Difference Equations, vol. 2011, Article ID 107384, 2011.

[12] M. Benchohra, S. Hamani, and S. K. Ntouyas, "Boundary value problems for differential equations with fractional order," Surveys in Mathematics and its Applications, vol. 3, pp. 1-12, 2008.

[13] Z. Bai and H. Lü, "Positive solutions for boundary value problem of nonlinear fractional differential equation," Journal of Mathematical Analysis and Applications, vol. 311, no. 2, pp. 495-505, 2005.

[14] M. Feng, X. Liu, and H. Feng, “The existence of positive solution to a nonlinear fractional differential equation with integral boundary conditions," Advances in Difference Equations, vol. 2011, Article ID 546038, 14 pages, 2011.

[15] Z.-W. Lv, J. Liang, and T.-J. Xiao, "Solutions to fractional differential equations with nonlocal initial condition in Banach spaces," Advances in Difference Equations, vol. 2010, Article ID 340349, 2010.

[16] S. Momani, A. Jameel, and S. Al-Azawi, "Local and global uniqueness theorems on fractional integro-differential equations via Bihari's and Gronwall's inequalities," Soochow Journal of Mathematics, vol. 33, no. 4, pp. 619-627, 2007.

[17] S. Dixit, O. Singh, and S. Kumar, "An analytic algorithm for solving system of fractional differential equations," Journal of Modern Methods in Numerical Mathematics, vol. 1, no. 1, pp. 1226, 2010 .

[18] A. Kilbas, H. Srivastava, and J. Trujillo, Theory and Applications of Fractional Differential Equations, Elsevier, Amsterdam, The Netherlands, 2006. 


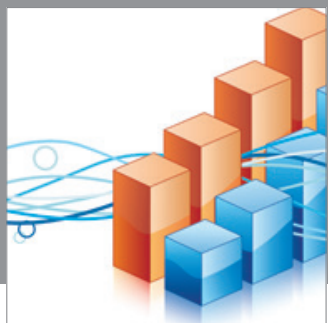

Advances in

Operations Research

mansans

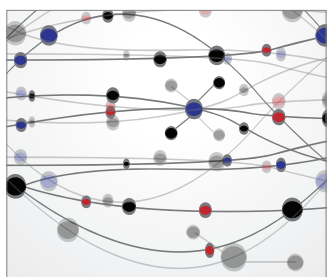

The Scientific World Journal
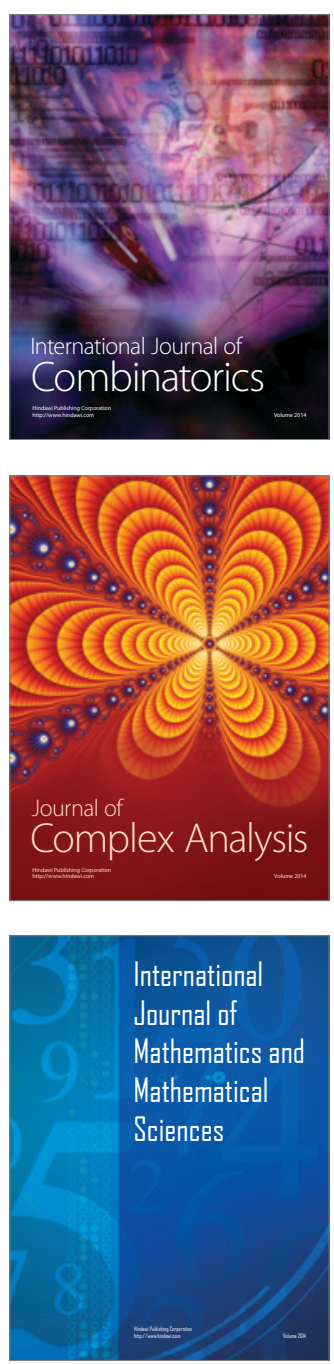
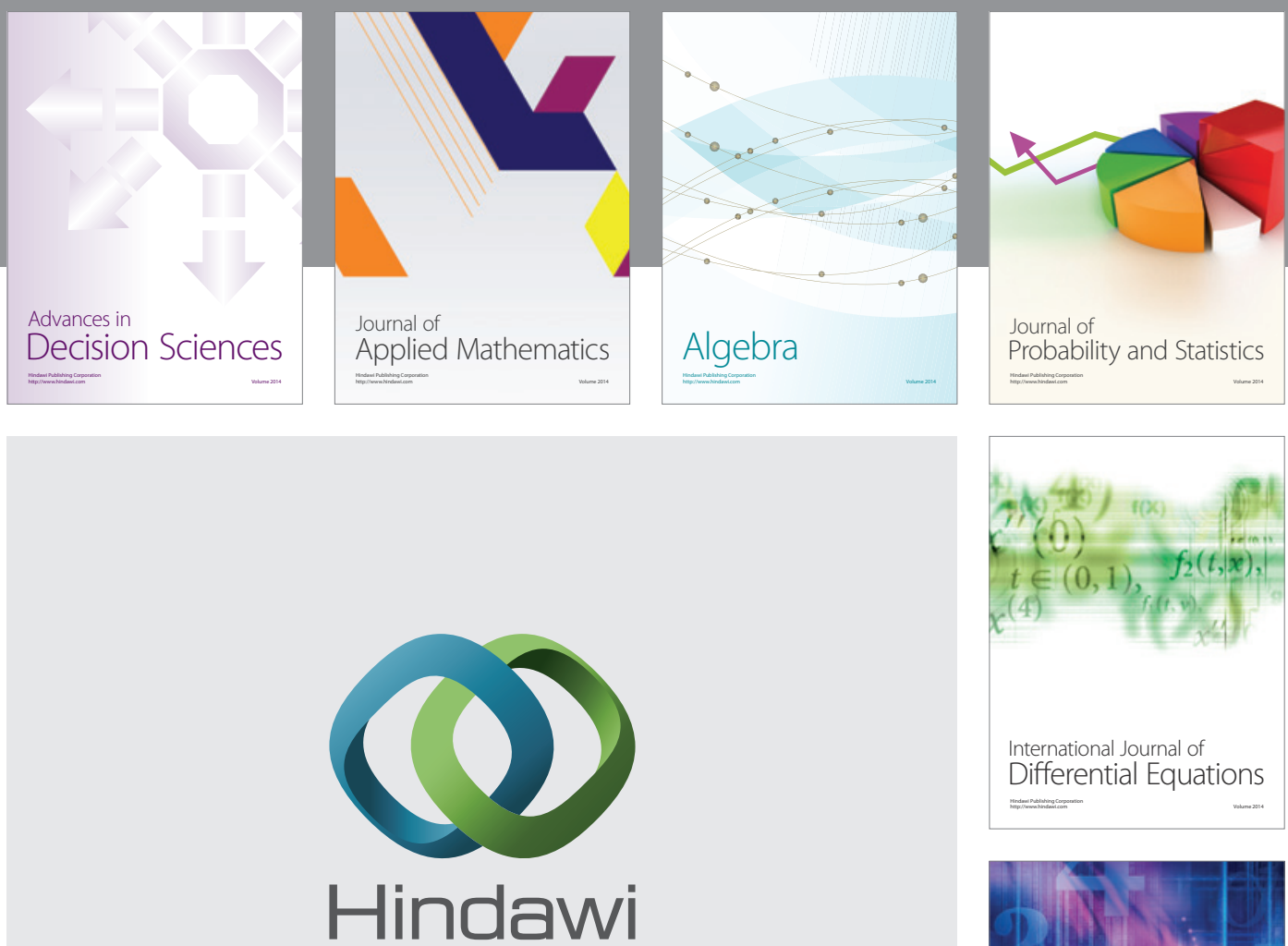

Submit your manuscripts at http://www.hindawi.com
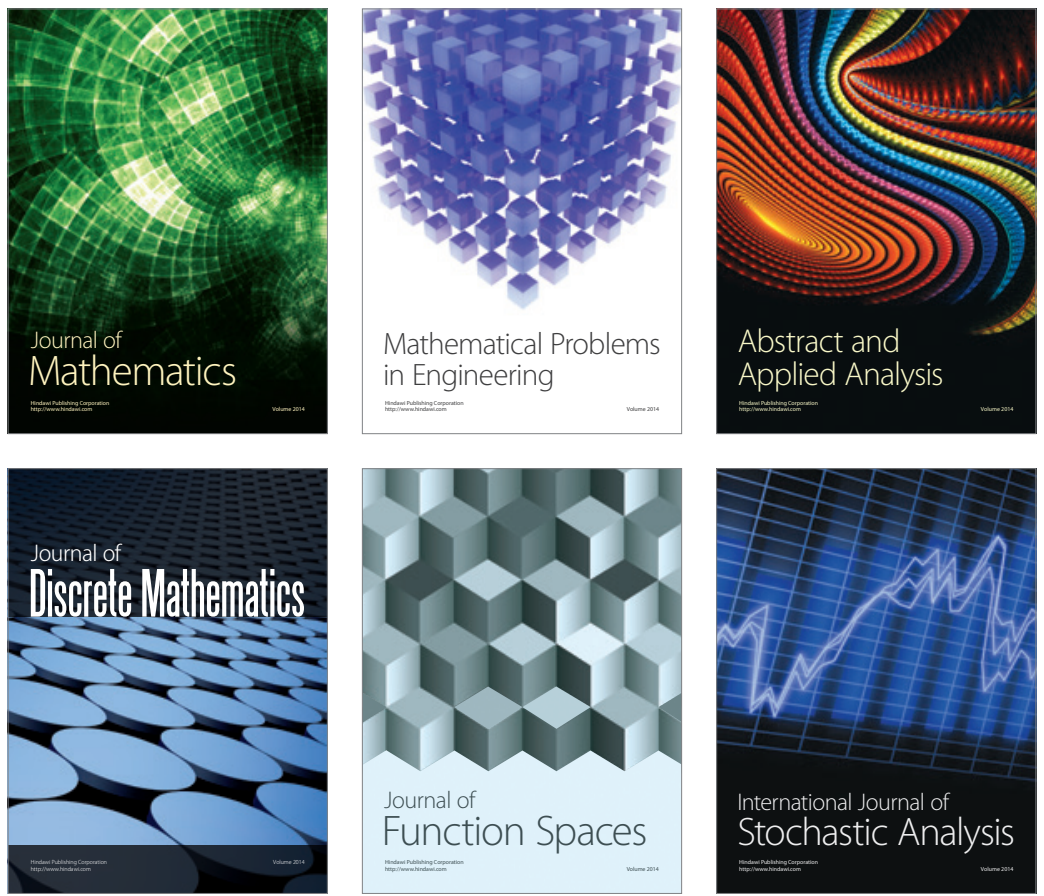

Journal of

Function Spaces

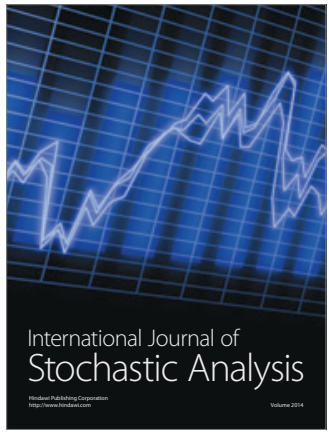

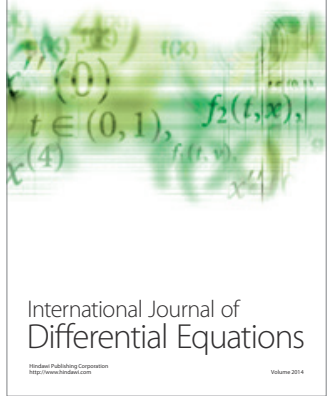
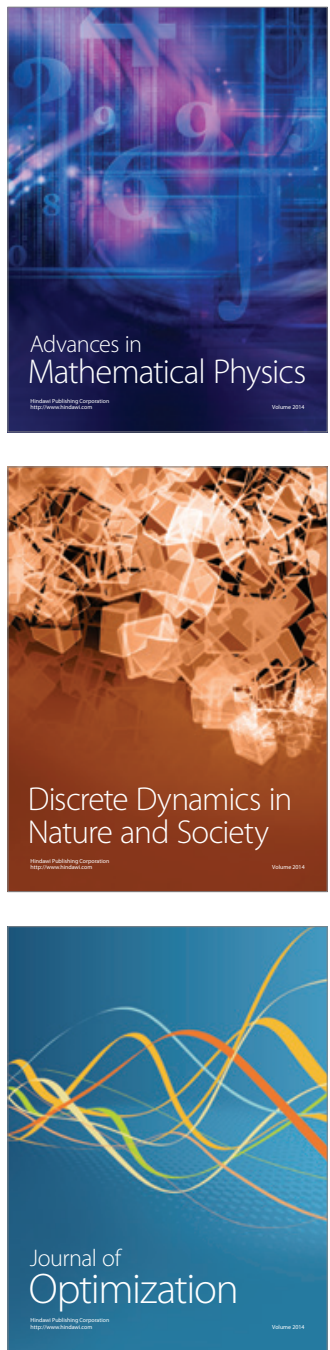